\title{
ASO Author Reflections: Significance of Skeletal Muscle Loss After Esophagectomy for Esophageal Cancer
}

\author{
Yuichiro Nakashima, MD, $\mathrm{PhD}^{1}$, and Masaki Mori, MD, PhD, FACS ${ }^{1}$ \\ Department of Surgery and Science, Graduate School of Medical Sciences, Kyushu University, Fukuoka, Japan
}

\section{PAST}

Esophagectomy and subsequent reconstruction with gastric conduit creation are associated with a risk of postoperative malnutrition via dysphagia due to anastomosis stricture, gastroesophageal reflux, gastric volume reduction, delayed food intake, impaired gastric emptying, and dumping symptoms. ${ }^{1}$ Previous studies have focused on the impact of baseline measures of skeletal muscle mass, obtained before surgery, on postoperative complications, prognosis, and treatment outcomes. ${ }^{2,3}$ However, to date, decreased skeletal muscle mass after esophagectomy for patients with esophageal cancer and its impact on postoperative long-term prognosis have not been fully investigated.

\section{PRESENT}

The current study ${ }^{4}$ is the largest retrospective study $(n=218)$ to focus on postoperative changes in skeletal muscle after esophagectomy for patients with esophageal cancer. Postoperative severe loss of skeletal muscle observed 6 months after esophagectomy was significantly associated with poor survival of esophageal cancer

ASO Author Reflections offer a brief invited commentary on the article, Skeletal Muscle Loss After Esophagectomy Is an Independent Risk Factor for Patients With Esophageal Cancer. Ann Surg Oncol. 2019. https://doi.org/10.1245/s10434-019-07850-6.

(C) Society of Surgical Oncology 2019

First Received: 15 October 2019;

Published Online: 21 November 2019

Y. Nakashima, MD, PhD

e-mail: yuicnakashima@gmail.com patients. Stratification showed that this association also occurred for patients with preoperative sarcopenia but not for patients without preoperative sarcopenia.

\section{FUTURE}

The current study did not measure other sarcopenic parameters on muscle quality, such as grip strength and walking speed. In the future, muscle quality data should be analyzed together with muscle quantity data. Exercise and nutritional support interventions may improve not only postoperative quality of life, but also long-term survival, particularly for esophageal cancer patients with preoperative sarcopenia.

DISCLOSURE The authors have no conflict of interest to disclose.

\section{REFERENCES}

1. Baker M, Halliday V, Williams RN, Bowrey DJ. A systematic review of the nutritional consequences of esophagectomy. Clin Nutr. 2016;35(5):987-94.

2. Nakashima Y, Saeki H, Nakanishi R, et al. Assessment of sarcopenia as a predictor of poor outcomes after esophagectomy in elderly patients with esophageal cancer. Ann Surg. 2018; 267(6):1100-4.

3. Ida S, Watanabe M, Yoshida N, et al. Sarcopenia is a predictor of postoperative respiratory complications in patients with esophageal cancer. Ann Surg Oncol. 2015;22(13):4432-7.

4. Nakashima Y, Saeki H, Hu Q, et al. Skeletal muscle loss after esophagectomy is an independent risk factor for patients with esophageal cancer. Ann Surg Oncol. 2019. https://doi.org/10.1245/ s10434-019-07850-6.

Publisher's Note Springer Nature remains neutral with regard to jurisdictional claims in published maps and institutional affiliations. 